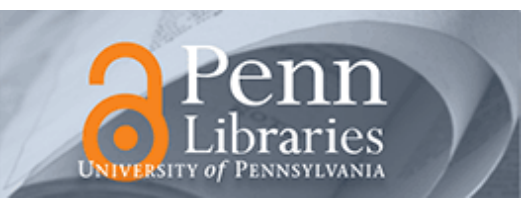

University of Pennsylvania

ScholarlyCommons

Departmental Papers (ESE)

Department of Electrical \& Systems Engineering

4-3-2009

\title{
A high fidelity ungrounded torque feedback device: The iTorqU 2.0
}

Kyle N. Winfree

University of Pennsylvania, winfree@udel.edu

Jamie E. Gewirtz

University of Pennsylvania, gewirtzj@seas.upenn.edu

Thomas Mather

University of Pennsylvania, mathert@seas.upenn.edu

Jonathan Fiene

University of Pennsylvania, jfiene@seas.upenn.edu

Katherine J. Kuchenbecker

University of Pennsylvania, kuchenbe@seas.upenn.edu

Follow this and additional works at: https://repository.upenn.edu/ese_papers

\section{Recommended Citation}

Kyle N. Winfree, Jamie E. Gewirtz, Thomas Mather, Jonathan Fiene, and Katherine J. Kuchenbecker, "A high fidelity ungrounded torque feedback device: The iTorqU 2.0", . April 2009.

Copyright YEAR 2009. Reprinted from:

Winfree, K.N.; Gewirtz, J.; Mather, T.; Fiene, J.; Kuchenbecker, K.J., "A high fidelity ungrounded torque feedback device: The iTorqU 2.0," EuroHaptics conference, 2009 and Symposium on Haptic Interfaces for Virtual Environment and Teleoperator Systems. World Haptics 2009. Third Joint, vol., no., pp.261-266, 18-20 March 2009 URL: http://ieeexplore.iee.. rg/stamp/stamp.jsp?arnumber=4810866\&isnumber=4810789

This material is posted here with permission of the IEEE. Such permission of the IEEE does not in any way imply IEEE endorsement of any of the University of Pennsylvania's products or services. Internal or personal use of this material is permitted. However, permission to reprint/republish this material for advertising or promotional purposes or for creating new collective works for resale or redistribution must be obtained from the IEEE by writing to pubs-permissions@ieee.org. By choosing to view this document, you agree to all provisions of the copyright laws protecting it.

This paper is posted at ScholarlyCommons. https://repository.upenn.edu/ese_papers/497

For more information, please contact repository@pobox.upenn.edu. 


\title{
A high fidelity ungrounded torque feedback device: The iTorqU 2.0
}

\author{
Abstract \\ This paper discusses the design and operation of the iTorqU 2.0, an ungrounded, handheld torque \\ feedback device for haptic applications. Based upon the gyroscopic effect, the iTorqU 2.0 uses a metal \\ flywheel inside of a two-axis actuated gimbal to create directional torques that are applied to the user's \\ hand. The coupling of angular velocity and angular momentum creates a torque that is orthogonal to the \\ two input angular velocities, giving the user the impression that their hand is being twisted in free air. \\ Following a review of prior work in the field of ungrounded torque feedback devices, we first present our \\ preliminary prototype, the iTorqU 1.0. Building on empirical observations and user feedback from a public \\ demonstration, we revised and augmented this design to create the iTorqU 2.0. This paper covers the \\ major mechanical, electrical, and controls design considerations that went into creating the iTorqU 2.0, \\ along with an analysis of its torque output capabilities.
}

\section{Keywords}

feedback, haptic interfaces, torque, angular momentum, angular velocity, haptic applications, iTorqU 2.0, ungrounded torque feedback device

\section{Comments}

Copyright YEAR 2009. Reprinted from:

Winfree, K.N.; Gewirtz, J.; Mather, T.; Fiene, J.; Kuchenbecker, K.J., "A high fidelity ungrounded torque feedback device: The iTorqU 2.0," EuroHaptics conference, 2009 and Symposium on Haptic Interfaces for Virtual Environment and Teleoperator Systems. World Haptics 2009. Third Joint, vol., no., pp.261-266, 18-20 March 2009

URL: http://ieeexplore.ieee.org/stamp/stamp.jsp?arnumber=4810866\&isnumber=4810789

This material is posted here with permission of the IEEE. Such permission of the IEEE does not in any way imply IEEE endorsement of any of the University of Pennsylvania's products or services. Internal or personal use of this material is permitted. However, permission to reprint/republish this material for advertising or promotional purposes or for creating new collective works for resale or redistribution must be obtained from the IEEE by writing to pubs-permissions@ieee.org. By choosing to view this document, you agree to all provisions of the copyright laws protecting it. 


\title{
A High Fidelity Ungrounded Torque Feedback Device: The iTorqU 2.0
}

\author{
Kyle N. Winfree* Jamie Gewirtz ${ }^{\dagger} \quad$ Thomas Mather $^{\ddagger} \quad$ Jonathan Fiene ${ }^{\S} \quad$ Katherine J. Kuchenbecker ${ }^{\llbracket}$ \\ Haptics Group, GRASP Laboratory \\ University of Pennsylvania, USA
}

\begin{abstract}
This paper discusses the design and operation of the iTorqU 2.0, an ungrounded, handheld torque feedback device for haptic applications. Based upon the gyroscopic effect, the iTorqU 2.0 uses a metal flywheel inside of a two-axis actuated gimbal to create directional torques that are applied to the user's hand. The coupling of angular velocity and angular momentum creates a torque that is orthogonal to the two input angular velocities, giving the user the impression that their hand is being twisted in free air. Following a review of prior work in the field of ungrounded torque feedback devices, we first present our preliminary prototype, the iTorqU 1.0. Building on empirical observations and user feedback from a public demonstration, we revised and augmented this design to create the iTorqU 2.0. This paper covers the major mechanical, electrical, and controls design considerations that went into creating the iTorqU 2.0, along with an analysis of its torque output capabilities.
\end{abstract}

\section{INTRODUCTION}

Haptic devices interact with a human user through the sense of touch, typically by changing positions, orientations, forces, torques, and/or vibrations. Such systems enable the user to receive rich streams of information from virtual or distant environments and to feel synthesized haptic sensations that resemble or deviate from those encountered in everyday life. Many different haptic interfaces have been developed, but the multi-channel nature of the sense of touch [7] provides ample inspiration for the invention of new devices and the exploration of new applications.

To be perceived as a mechanical influence, a haptic device must act on the user with forces and/or torques, which always occur in equal and opposite pairs. Thus, one can classify haptic interfaces into three categories based on their mechanical grounding configuration [2]. The classically grounded device is one that rests on a table, floor, or other static object that can be used as leverage. Typically constructed as mechanical linkages with rotational and translational joints, these devices can thus employ actuators or brakes to impose forces and/or torques between the user and the grounding element. Capable of producing salient haptic sensations with relatively low mechanical complexity, grounded devices are well represented in the commercial market, with systems produced by companies such as SensAble Technologies, MPB Technologies, and FCS Control Technologies, among others [5]. Unfortunately, grounded haptic devices also suffer from finite workspace constraints and a continual need to reduce inertial and friction effects to accurately render free-space motion [4].

The second major category of haptic devices is that of body grounded systems. These interfaces are affixed to part of the user's

\footnotetext{
*e-mail: winfree@seas.upenn.edu

†e-mail:gewirtzj@seas.upenn.edu

‡e-mail:mathert@seas.upenn.edu

$\S$ e-mail:jfiene@seas.upenn.edu

ฯe-mail:kuchenbe@seas.upenn.edu
}

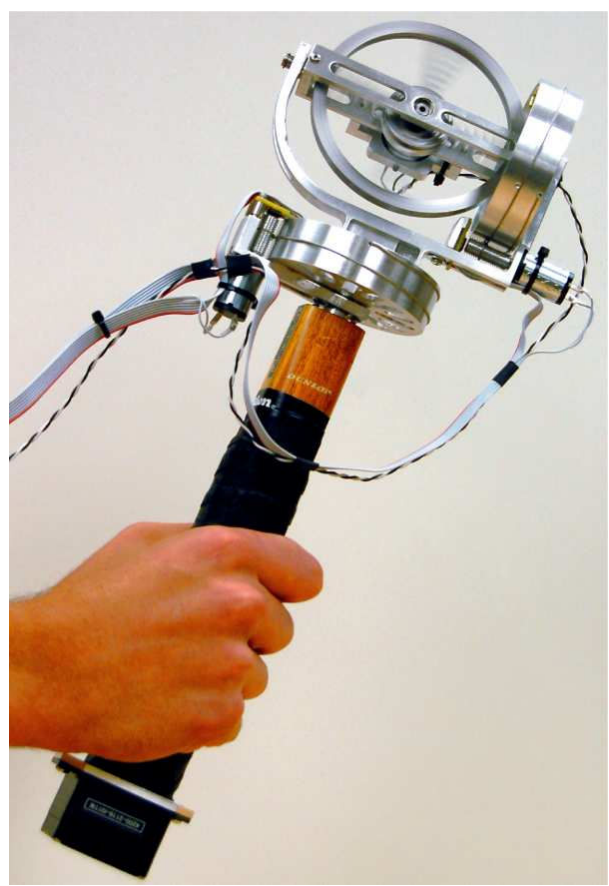

Figure 1: The iTorqU 2.0 in use.

body rather than to an immobile object. The device uses this body mount as the leverage point for applying equal and opposite forces and/or torques to another part of the wearer's body. By carefully considering how the grounding forces are positioned and distributed, such devices can create the illusion of directed forces [9]. One such device is Immersion Corporation's CyberGrasp, in which forces applied to the user's fingers are grounded to the forearm; this design takes advantage of the fact that fingertip mechanoreceptors are significantly more sensitive than those of the forearm [7]. Such devices are generally more portable than grounded displays, but they can be challenging to design due to strict objectives for strength, weight, and ergonomics.

The third category of haptic devices includes those that are mechanically ungrounded. Often employing linear or angular momentum to create an inertial ground, these devices need not be attached to a table or one's body to generate the intended interaction loads; thus, they can be highly portable. One such example of ungrounded haptic feedback can be found in mobile phones and modern video game controllers [6]. Though these devices excel at rendering cyclical vibrations, they are incapable of generating discernible directed forces or torques. Over the past decade, several research groups have explored novel methods of producing ungrounded directed force feedback, such as air jets [3] and asymmetric oscillation [1], but only a few have investigated ungrounded directed torque output, which has exciting potential applications in interactive gaming, navigational guidance, and upper-limb rehabilitation. Section 2 describes prior haptic devices in this area, and the remainder of the paper presents the development of a new, high fidelity, ungrounded torque feedback device known as the iTorqU 2.0. 


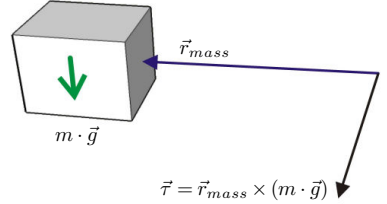

(a) Force at a distance

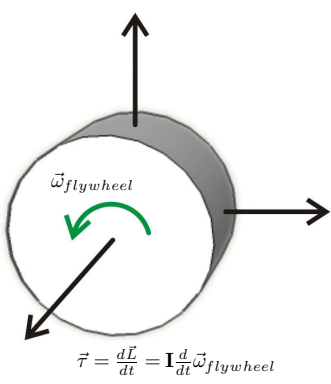

(b) Momentum wheel

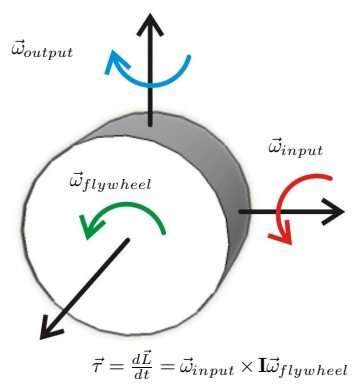

(c) Steered momentum wheel

Figure 2: Various methods by which a haptic device can create ungrounded torque feedback using mass and inertia. (a) Torque by $\vec{F}_{\text {gravity }}$ at a distance. This effect is used in the TorqueBAR. (b) Axes of actuation and output of the spinning mass momentum wheel. $\dot{\vec{\omega}}_{f l y w h e e l}$ is the angular acceleration of input. This effect is used in the GyroCube. (c) Axes of actuation and output of the steered spinning mass. $\vec{\omega}_{i n p u t}$ is the angular velocity of input, $\vec{\omega}_{\text {flywheel }}$ is the angular velocity of the flywheel, and $\vec{\omega}_{\text {output }}$ is the output angular velocity. This effect is used in the Gyro Moment Display and the iTorqU 1.0 and 2.0.

\section{Prior art in Ungrounded Torque Feedback}

Exploring a variety of different actuation methods, significant developments in the field of ungrounded torque output haptic devices include the TorqueBAR [8], the GyroCube [10], and the Gyro Moment Display [11], each of which will be described below.

The TorqueBAR [8] is a single-degree-of-freedom system that generates moments about a set of handles by moving a large mass along a linear slide, as shown in Figure $2 \mathrm{a}$. The linear slide on this device is 48-cm long; an accelerometer is used to measure the tilt angle. In a series of human-subject tests, the authors were able to show that torque feedback aided subjects in performing a simple position trajectory-matching task.

As an alternative mode of actuation, the GyroCube [10] is a handheld device containing three fixed-axis, orthogonal flywheels that can be positively or negatively accelerated to generate torques about the major axes, as shown in Figure 2b. Unfortunately, experimental results found that users had difficulty distinguishing the direction of the generated torques, most likely due to gyroscopic effects (i.e., because all three wheels were spinning when the angular momentum of one was changed, the other two may have acted as steered momentum wheels, thereby creating unintended gyroscopic moments that interfered with the intended torque output).

The Gyro Moment Display [11] capitalizes on the gyroscopic effect by mounting a rotating disk in a two-axis gimbal. By controlling the angular rate of the gimbal axes, the device is capable of generating torques orthogonal to the rotational axis of the flywheel, as shown in Figure 2c. One significant benefit of using controlledrate gyros (as opposed to momentum wheels) is that when the inertia and speed of the flywheel are both relatively large, small changes in the gimbal rates result in significant output torques. While the device is capable of producing arbitrary ungrounded torques, the authors noted that with a relatively small range of gimbal travel $\left( \pm 50^{\circ}\right.$ for each axis), the Gyro Moment Display required a significant amount of time to return the gyroscope to a centered configuration before display of an additional moment, thereby severely limiting the device bandwidth. The authors did not sense orientation of the device relative to ground. Given the unknown pose and high back drive friction, users would inadvertently feel gyroscopic moments when the pose of the device was perturbed.

\section{First Prototype: The ITORQU 1.0}

Taking inspiration from the prior art in ungrounded torque feedback, we developed a single-axis proof-of-concept device in the spring of 2008. As shown in Figure 3, this version included a

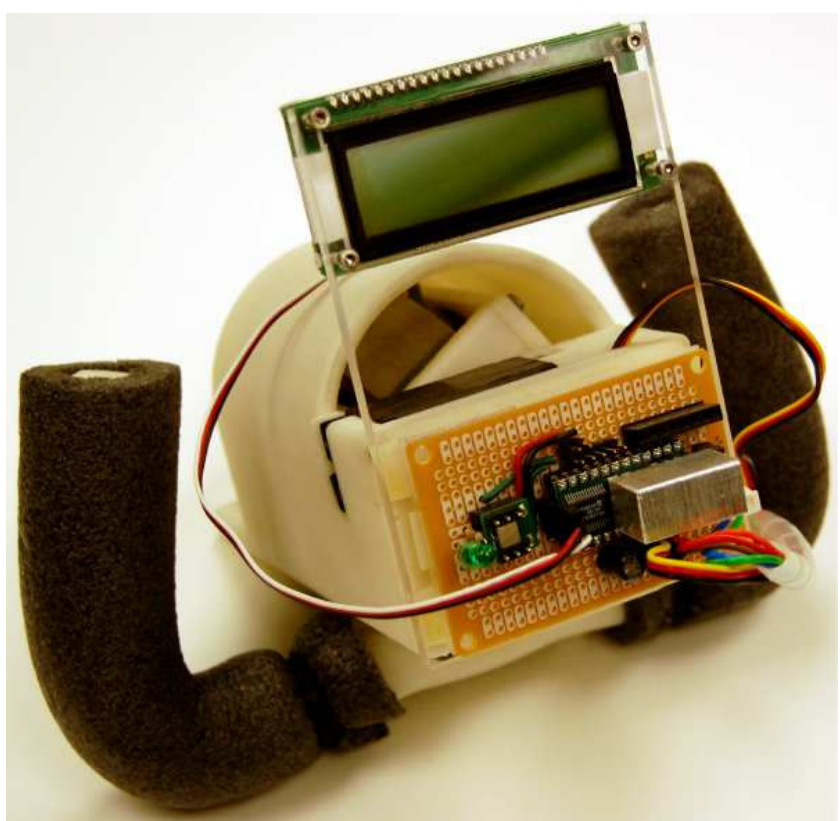

Figure 3: The iTorqU 1.0

steel flywheel (shown in Figure 4) mounted within a single gimbal driven by a hobby servo. The system was able to display ungrounded torques by driving the gimbal at a given rate. A two-axis accelerometer was used to resolve the spatial orientation of the device relative to ground. This pose estimation was used to make the device pseudo-transparent; when the user slowly rotated the device about the gimbal axis, the flywheel was steered in the opposite direction. By maintaining a constant ground orientation of the flywheel, the device did not create the unintended output torques felt in [11]. This section presents the design and development of this prototype, with a focus on the lessons learned.

\subsection{Design}

Mechanical - For rapid prototyping, the housing, internal gimbal cage, and handles were all constructed using fused-depositionmodeling (FDM) from ABS plastic. Given the relatively low 


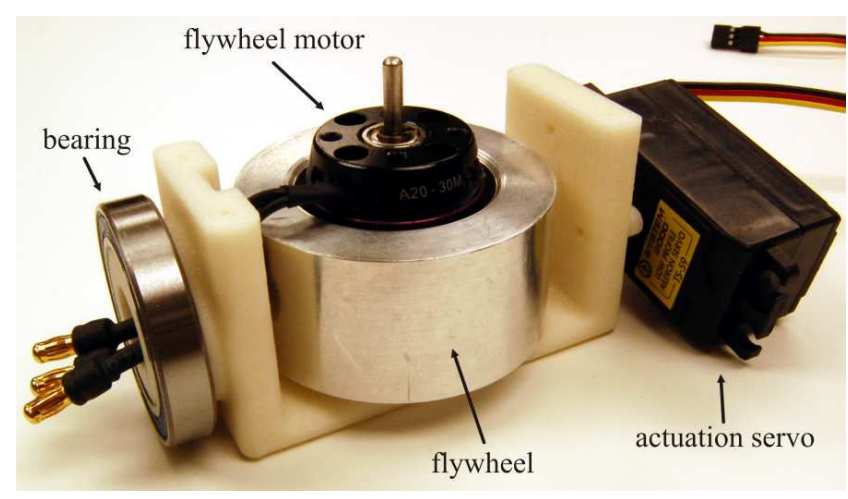

Figure 4: An excised view of the flywheel and drive train of the iTorqU 1.0.

strength of the plastic, the unit was designed with two handles for the user to grasp, as can be seen in Figure 5. To minimize complexity and development time, the system included only one gimbal axis which was oriented normal to the handles. Two flywheels were designed and tested, one from aluminum $(\mathrm{m}=95 \mathrm{~g}$, $\left.\mathrm{I}=37500 \mathrm{~g} \cdot \mathrm{mm}^{2}\right)$ and one from cold-rolled steel $(\mathrm{m}=281 \mathrm{~g}$, I $=111000 \mathrm{~g} \cdot \mathrm{mm}^{2}$ ), both of which have a mass radius of gyration $\left(k_{m}=\sqrt{\frac{I}{m}}\right)$ of $19.6 \mathrm{~mm}$.

Actuation - A brushless Hacker A20-30M hobby motor (9000 $R P M)$ was chosen for its ability to be recessed within the flywheel and its high torque and speed output. A Tower Hobbies TS-59 lowprofile servo motor was used to directly drive the gimbal axis using local closed-loop position control.

Sensing - A fundamental requirement for any ungrounded haptic feedback device is the ability to become transparent (i.e., to produce minimal feedback forces and torques when the user is moving in free space). In order to produce transparency with a gyroscope, the orientation of the spinning mass must remain constant relative to the ground reference frame. To accomplish this task with our device, we mounted a model 2125 Memsic Dual-Axis Accelerometer to the housing to measure the device's orientation relative to ground. By actively controlling the gimbal angle from the estimated device orientation, we were able to operate the device in a pseudo-transparent mode.

Software - A Parallax Basic Stamp 2 microcontroller was used to sample the accelerometer and implement the gimbal control system. When operating in transparency mode, the system continually commanded the gimbal servo to the opposite of the resolved device angle, thereby attempting to maintain the ground-reference flywheel orientation. A set of switches was also connected to the device to command the gimbal servo to either \pm 90 or \pm 45 degrees at either a slow or fast rate to generate ungrounded output moments. Due to the relatively limited range of motion for the gimbal servo $\left( \pm 120^{\circ}\right)$, the servo was slowly returned to a center position after each output at a speed below the noticeable output torque.

\subsection{Lessons Learned}

The iTorqU 1.0 was demonstrated at the University of Pennsylvania Haptics Laboratory Open House on May 1, 2008, where approximately fifty individuals from various backgrounds were able to test the device. Many users provided positive comments about the design, most notably that it produced strong output torques; some users also raised concerns about the weight and strength of the device. The overwhelmingly positive interest in the device motivated our construction of the second-generation model described in the following section. The major lessons learned, both from the design

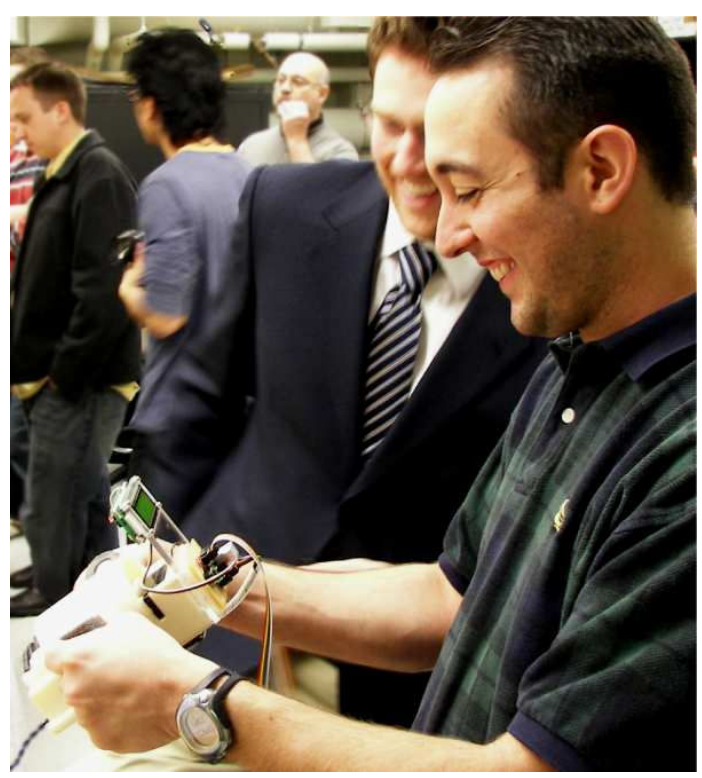

Figure 5: An open house attendee testing the iTorqU 1.0

process and from the informal user feedback can be summarized as follows:

Mechanical - While fused-deposition-modeling was useful for generating a quick prototype, the low stiffness of ABS was not suitable for a system generating sizable torques. More importantly, the unit was relatively heavy $(833 \mathrm{~g})$ compared with the discernible torque output. Specifically focusing on the torque source, we found that while the steel flywheel produced larger torque output, it also produced significantly more vibration and resulted in an overall heavier device. The original gimbal design severely limited the diameter of the flywheel, requiring an undesirable mass to moment-of-inertia ratio. We also learned that the quality of the bearings supporting the flywheel shaft can have a significant impact on the generation and transmission of unwanted vibrations throughout the system.

Actuation - While capable of high-speed operation, the brushless flywheel motor produced noticeable high-frequency vibrations. After inspection of many other hobby-type brushless motors, it was determined that this was not a motor-specific problem, but instead is common to the design of brushless hobby motors. Secondarily, while the gimbal servo motor was convenient, its internal positioncontrol loop prevented us from implementing feedback control of gimbal velocity. The internal gearing of the gimbal servo had high back drive friction, which made transparency significantly more difficult to achieve (in theory, a frictionless gimbal would allow the flywheel to maintain orientation regardless of the device angle, thereby creating natural transparency). We also discovered that the bandwidth of the gimbal servo was too low to produce the range of desired output torques, and the limited range of motion imposed unwanted constraints on the usable workspace of the device.

Sensing - Again, the lack of position feedback on the gimbal cage imposed a significant constraint on our ability to accurately drive the system transparently. We also discovered that the bandwidth of the filter used to remove flywheel induced noise on the dual-axis accelerometer prevented the accurate resolution of orientation for rapid handle movements, restraining users to relatively slow turns.

\section{The iTorou 2.0: System Design}

Building upon the success and many lessons learned from the preliminary prototype, we designed and built a dual-axis gimbal- 
controlled gyroscopic haptic display. Herein we will discuss the major components of the device, including the flywheel, the gimbals, the handle, and the control system.

\subsection{Flywheel}

To maximize the mass radius of gyration, the new flywheel was comprised of an annular ring with four thin spokes connected to a central hub, as can be seen in Figure 6a. The moment of inertia of the annular ring is given by

$$
I_{z z}=\frac{1}{2} m\left(r_{2}^{2}+r_{1}^{2}\right)=\frac{1}{2} \rho h \pi\left(r_{2}^{4}-r_{1}^{4}\right)
$$

where $m$ is the mass, $\rho$ is the density, $h$ is the height, $r_{1}$ is the inner radius, and $r_{2}$ is the outer radius (for our design: $h=5.25 \mathrm{~mm}$, $r_{1}=86 \mathrm{~mm}, r_{2}=99 \mathrm{~mm}$ ). The flywheel was machined from 416 stainless steel to a total mass of 138 grams and a calculated moment of inertia of $218000 \mathrm{~g} \cdot \mathrm{mm}^{2}$. This design resulted in a mass radius of gyration of $39.75 \mathrm{~mm}$, which is more than double that of the iTorqU 1.0 (in other words, for the same flywheel mass, the new design has four times more rotational inertia). To drive the flywheel, the brushless hobby motor was replaced with a Faulhaber MicroMo 2607T006SR pancake motor, with a no-load speed of $6600 R P M$ at $6 \mathrm{~V}$. With a mass of only $16.1 \mathrm{~g}$, this motor provided a smooth, high-velocity input to the flywheel with a minimum of added mass.

\subsection{Gimbals}

One of the most significant changes in this version of the iTorqU was the addition of a second gimbal axis, as shown in Figure 6a, to enable the creation of full three-DOF torques and allow transparency in all directions. While adding this second degree of freedom, we also wanted to significantly extend the angular workspace of each gimbal. The gimbal cages were custom machined from 6061-T6 aluminum to achieve low mass while maintaining relatively high stiffness. To minimize joint friction, precision Dynaroll ABEC 7 oil-lubricated bearings were used for all rotary joints.

While mounting motors directly to the gimbal axes would reduce mechanical complexity and could theoretically provide an infinite angular workspace, the weight and size of the motors and gearheads necessary to create the gimbal torques made such a solution impractical. In addition, the reduced backdriveability of the gearheads would make transparency more difficult. As an alternative, custom drum-and-capstan cable drive systems were designed for each axis. Powered by Maxon 110045 brushed DC motors (mass of $21 \mathrm{~g}$ and a stall torque of $4.78 \mathrm{mNm}$ at $12 \mathrm{~V}$ ) and cabled with Sava Industries 2032 stainless-steel cable, the completed gimbal drivetrains can be seen in Figure 6c. With very low friction, smooth backdriveability, and a gear ratio of 10.2 , the final configuration resulted in a $540^{\circ}$ range of motion for each axis. When fully assembled with the flywheel, the mass of the full two-axis gimballed gyroscope was $486 \mathrm{~g}$.

To accurately measure the gimbal axis angles, diametric magnets were attached to the end of each capstan, and AS5040 10-bit programmable hall-effect rotary encoders were located in the middle of the rotating magnetic fields, as can be seen in Figure 6c. With an angular resolution of $0.3^{\circ}$, and assuming no slip in the cable drive system, this arrangement provided angular displacement data for each gimbal axis down to $0.032^{\circ}$.

\subsection{Handle}

To create a naturally affordant user interface, a wooden tennis racket handle, with an additional mass of $248 \mathrm{~g}$, was affixed to the base of the device, as seen in Figure 1. This single-handed configuration was chosen so that the torques outputted from the device would be more noticeable to the user. To resolve the ground-referenced angular configuration of the system, a MicroStrain 3DM-GX2-USB-SK1 inertial-measurement unit (IMU) was

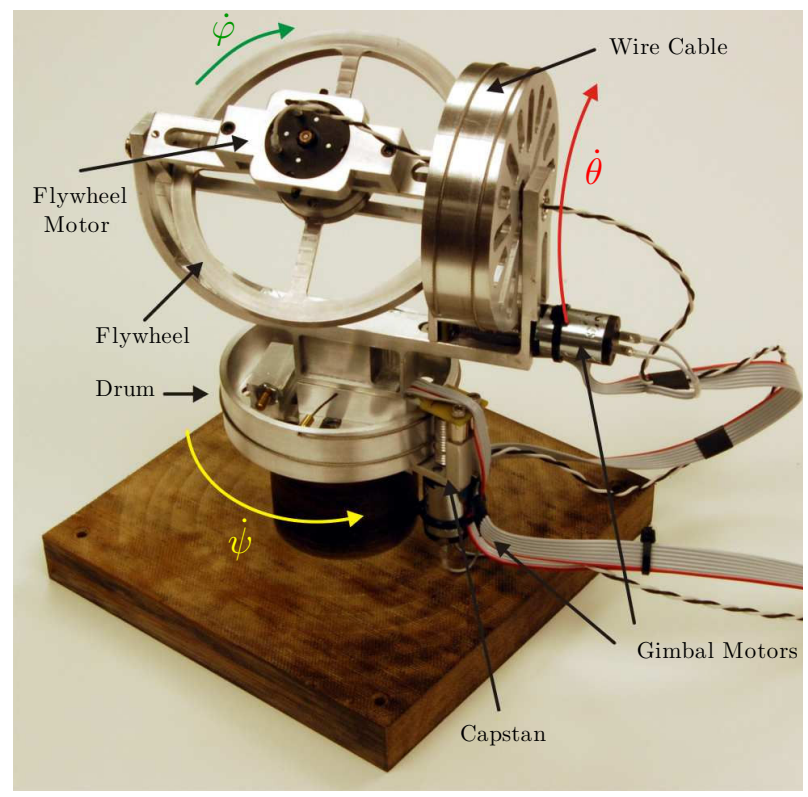

(a) The axes and components of the iTorqU 2.0. Shown here, it is ready to mount to the AMTI force-torque plate.
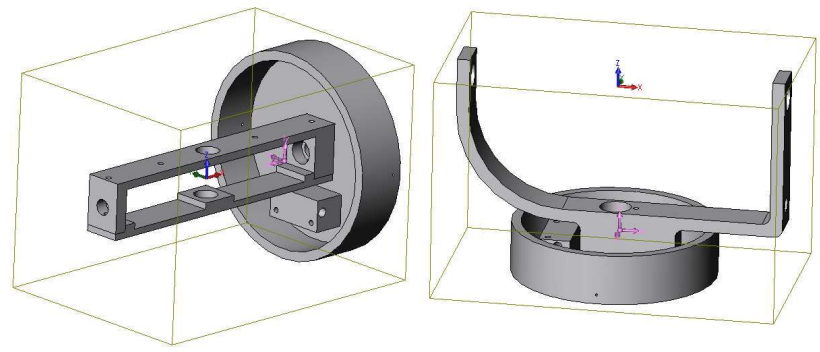

(b) A CAD model of the top (left) and bottom (right) gimbals used in the iTorqU 2.0.

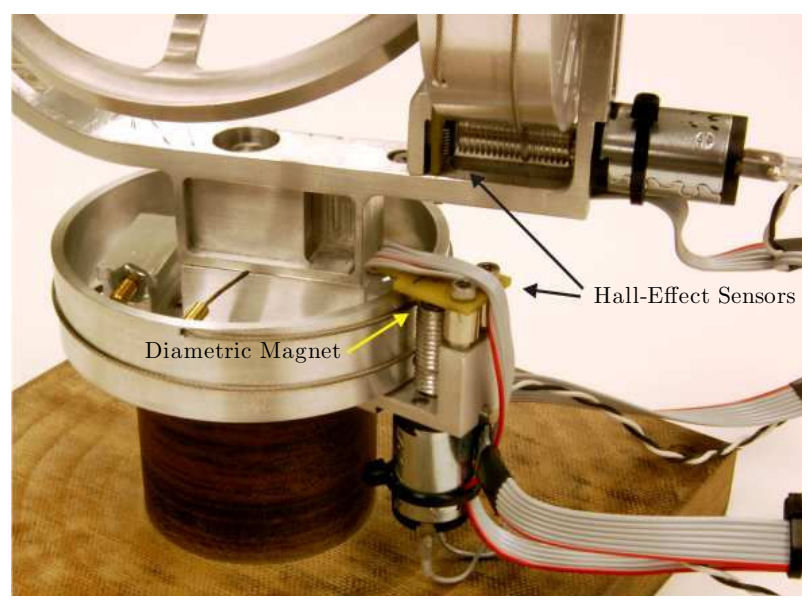

(c) The drum-and-capstan cable drives for both gimbal axes. The diametric magnet is embedded in the aluminum capstan.

Figure 6 
attached to the base of the handle. With a mass of only $49 \mathrm{~g}$, this compact IMU combines three degrees of acceleration sensing with three rate gyros and a magnetometer to provide absolute orientation measurements with a resolution of less than $0.1^{\circ}$ at a data rate of up to $250 \mathrm{~Hz}$.

\subsection{Controller}

Running on a Windows XP computer, Chai 3D was used to implement a control loop for the generation of three-dimensional output torques from the device. The handle-mounted IMU communicated directly over the USB port, while a PIC microprocessor was programmed to convert the SPI communications from the hall-effect encoders to USB signals. To control the gimbal motors, a Sensoray $626 \mathrm{PCI}$ interface board was used for analog output to a pair of OPA544T high-current operation amplifiers; this arrangement enabled high-bandwidth closed-loop current control for each axis.

\section{Modeling Output Torques}

The moments generated by the iTorqU can be modeled by summing the moments generated by a rotating body spun around another axis (the flywheel), plus the moments caused by rotational acceleration of the gimbals (inertia), plus the moments from gravity (a force at a distance). These three items include all three effects used by the various authors mentioned in section 2 .

This model can then be used to predict the output moments for given state variables $(\psi, \theta, \dot{\psi}, \dot{\theta})$ and input variables $(\ddot{\psi}, \ddot{\theta})$ as is shown later in Figure 7d.

The moments caused by the flywheel, in the frame of the flywheel, are:

$$
{ }^{\theta} \vec{M}_{\text {flywheel }}=\mathbf{I} \cdot \dot{\vec{\omega}}_{\text {system }}+\vec{\omega}_{\text {frame }} \times\left(\mathbf{I} \cdot \vec{\omega}_{\text {system }}\right)
$$

Then rotating these moments to a ground frame:

$$
{ }^{g} \vec{M}_{\text {flywheel }}={ }^{g} \mathbf{R}_{\theta}{ }^{\theta} \vec{M}_{\text {flywheel }}
$$

where ${ }^{g} \mathbf{R}_{\theta}$ includes a rotation from the $\theta$ frame to a handle frame and then a rotation from the handle frame to the ground frame.

The moments from the acceleration of the gimbals then is:

$$
\begin{aligned}
{ }^{g} \vec{M}_{\text {top } \ddot{\theta}, \ddot{\psi}} & ={ }^{g} \mathbf{R}_{\psi} \cdot\left(\mathbf{I}_{\text {top }} \cdot \ddot{\theta}\right) \\
{ }^{g} \vec{M}_{\text {bottom } \ddot{\psi}} & ={ }^{g} \mathbf{R}_{h} \cdot\left(\mathbf{I}_{\text {bottom }} \cdot \ddot{\psi}\right)
\end{aligned}
$$

Finally, the moments due to gravity are:

$$
\begin{aligned}
{ }^{g} \vec{M}_{\text {top gravity }} & =\left({ }^{g} \mathbf{R}_{\theta} \cdot{ }^{\theta} \vec{r}_{\text {top cm }}\right) \times \vec{F}_{\text {gravity }} \\
{ }^{g} \vec{M}_{\text {bottom gravity }} & =\left({ }^{g} \mathbf{R}_{\psi} \cdot{ }^{\psi} \vec{r}_{\text {bottom cm }}\right) \times \vec{F}_{\text {gravity }}
\end{aligned}
$$

where $\vec{r}_{* \mathrm{~cm}}$ is the vector pointing to the center of mass of that gimbal.

This sum, the torque a user would feel while using the iTorqU 2.0, is then:

$$
\begin{aligned}
{ }^{g} \vec{M}_{\text {total }}= & { }^{g} \vec{M}_{\text {flywheel }}+{ }^{g} \vec{M}_{\text {top } \ddot{\theta}, \ddot{\psi}}+{ }^{g} \vec{M}_{\text {bottom } \ddot{\psi}}+ \\
& { }^{g} \vec{M}_{\text {top gravity }}+{ }^{g} \vec{M}_{\text {bottom gravity }}
\end{aligned}
$$

\section{Operation and Testing}

To validate the performance characteristics of the iTorqU 2.0, a series of calibration tests was performed with the device attached to an AMTI HE6X6-10-5 6-DOF grounded force/torque plate. A custom adapter was used to mount the iTorqU to the center of the plate with the base gimbal axis aligned with the plate's $\mathrm{Z}$ axis. Data was recorded at a rate of $200 \mathrm{~Hz}$, with resolutions of $0.07 \mathrm{mN}$ for the principal forces and $0.03 \mathrm{mN} \cdot \mathrm{m}$ for the principal torques. To analyze the effect of independently gimbaling each axis, one of the

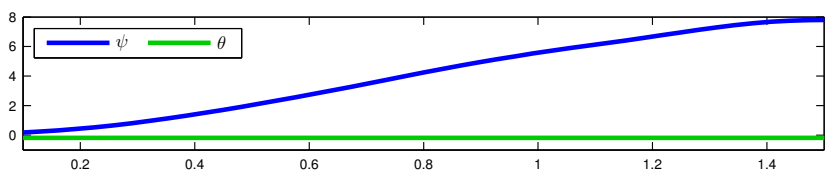

(a) Measured angles ( $\mathrm{rad}$ ) vs. time (s).

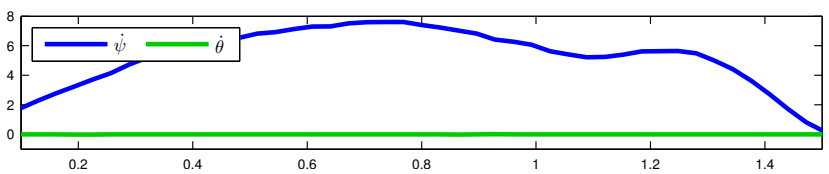

(b) Measured velocities $(\mathrm{rad} / \mathrm{s})$ vs. time $(\mathrm{s})$.

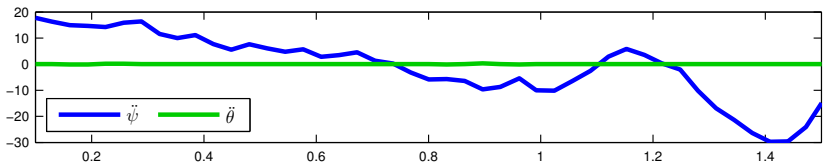

(c) Measured accelerations $\left(\mathrm{rad} / \mathrm{s}^{2}\right)$ vs. time $(\mathrm{s})$.

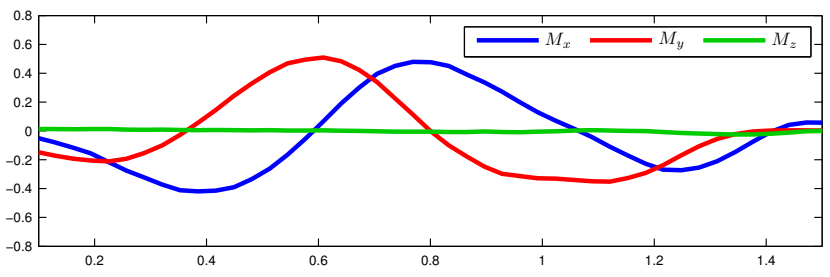

(d) Predicted moments $(m N \cdot m)$ vs. time $(s)$

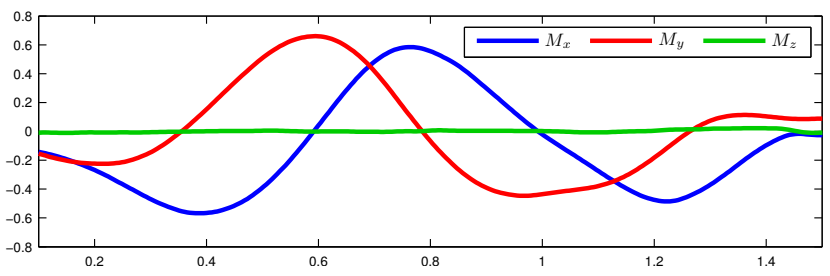

(e) Measured moments $(m N \cdot m)$ vs. time $(s)$.

Figure 7: For this trial, the yaw gimbal $(\psi)$ was rotated through its range of motion (applied motor voltage of 6.2V) while the pitch gimbal was constrained. As can be seen in comparing (d) and (e), the measured moments are very close to the predicted moments. Possible reasons for error may include accuracy of modeled mechanical and electrical components.

two gimbals was physically constrained while the other was actuated through its range of motion at a variety of speeds.

Figure 7 shows samples of the recorded angles $7 \mathrm{a}$, angular velocities $7 \mathrm{~b}$, angular accelerations $7 \mathrm{c}$, and torque data $7 \mathrm{e}$ as the base (yaw) gimbal was spun through its full range of motion while the upper (pitch) gimbal was constrained to keep the flywheel axis horizontal. Figure $7 \mathrm{~d}$ shows the expected moments from the motions of the gimbals and flywheel as predicted with our model (6).

Figure 8a presents the magnitude and phase of the resultant moment in the $\mathrm{X}-\mathrm{Y}$ plane for four different yaw gimbal excitation voltages. Likewise, Figure $8 \mathrm{~b}$ presents the magnitude and phase of the moments created in the X-Z plane as the pitch gimbal is actuated while the yaw gimbal was constrained to keep the flywheel axis in the Y-Z plane. With a maximum torque for each independent gimbal axis of nearly $1.2 \mathrm{Nm}$ and workspaces that extend well beyond 360 degrees, this data is very encouraging for the development of a range of future applications. 


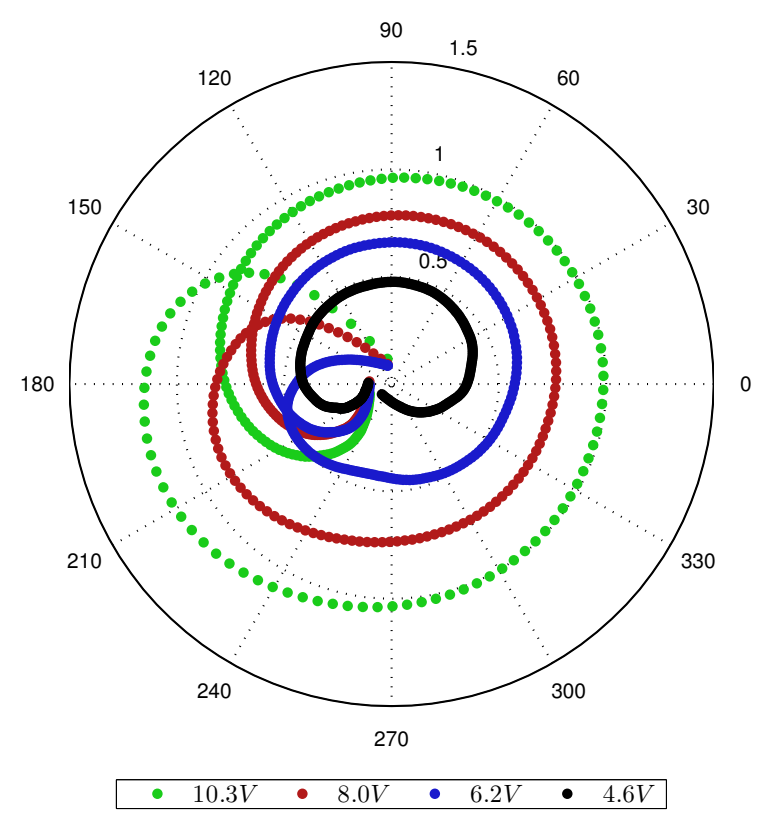

(a) Yaw gimbal $(\psi)$ was rotated with the pitch gimbal fixed. Moment in $\mathrm{X}-\mathrm{Y}$ plane.

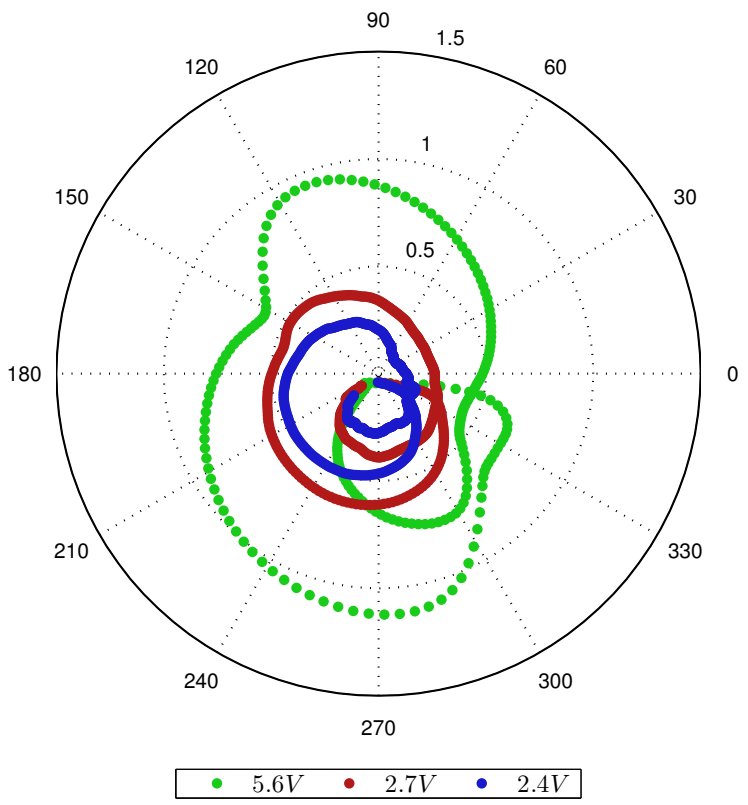

(b) Pitch gimbal $(\theta)$ was rotated with the yaw gimbal fixed. Moment in $\mathrm{X}-\mathrm{Z}$ plane.

Figure 8: The magnitude $(N \cdot m)$ and angle $\left(^{\circ}\right)$ of the moment vector.

\section{Conclusions and Future Work}

Through the development of testing of two successive versions of the iTorqU haptic device, we have proven the feasibility of using a gimbal-controlled gyroscope to generate ungrounded torque feedback. By optimizing the flywheel design, the mass radius of gyration of the second device was increased while concurrently reducing the overall mass such that it is comfortable to hold for extended durations. This second version was then able to create more sub- stantial torques while reducing user fatigue. The large workspace, low friction, and easy backdriveability of the two independent gimbal joints provide a high degree of natural transparency as the flywheel is free to maintain its orientation as the handle is moved below it. By actively controlling the angular velocity of the two gimbals, significant ungrounded torques can easily be produced at the handle, as demonstrated by the results presented in Section 6 .

With the hardware platform fully operational and preliminary tests showing favorable results, future work on this project will include the implementation of a full closed-loop control system to generate desired torques about arbitrary axes. A second controldevelopment project will be focused on the tuning and evaluation of an active-transparency mode to combine the data from the IMU and the hall-effect sensors to accurately estimate the handle and flywheel orientation and actively cancel out any remaining gyroscopic effects. Thought will then be given to modes for generation of desired torques using the model mentioned in section 5. Finally, a human-subject study will be conducted to quantify the effectiveness of the iTorqU 2.0 ungrounded torque output device in psychophysical tests and representative applications.

A demonstration of both the iTorqU 1.0 and iTorqU 2.0 will be provided at 2009 World Haptics Conference in Salt Lake City, Utah.

\section{REFERENCES}

[1] T. Amemiya and T. Maeda. Asymmetric oscillation distorts the perceived heaviness of handheld objects. IEEE Transactions on Haptics, 1(1):9-18, June 2008.

[2] S. J. Biggs and M. A. Srinivasan. Haptic interfaces. In K. Stanney, editor, Handbook of Virtual Environments: Design, Implementation, and Applications, Human Factors and Ergonomics, chapter 5, pages 93-115. Lawrence Erlbaum Associates, 2002.

[3] H. Gurocak, S. Jayaram, B. Parrish, and U. Jayaram. Weight sensation in virtual environments using a haptic device with air jets. ASME Journal of Computing and Information Science in Engineering, 3:130135, June 2003.

[4] V. Hayward and O. R. Astley. Performance measures for haptic interfaces. In G. Giralt and G. Hirzinger, editors, Robotics Research: The Seventh International Symposium, pages 195-207. Springer Verlag, 1996.

[5] V. Hayward, O. R. Astley, M. Cruz-Hernandez, D. Grant, and G. Robles-De-La-Torre. Haptic interfaces and devices. Sensor Review, 24(1):16-29, 2004.

[6] V. Hayward and K. E. MacLean. Do it yourself haptics: Part I. IEEE Robotics and Automation Magazine, pages 88-104, December 2007.

[7] R. L. Klatzky and S. J. Lederman. Touch. In A. F. Healy and R. W. Proctor, editors, Handbook of Psychology, volume 4: Experimental Psychology, chapter 6, pages 147-176. John Wiley and Sons, 2003.

[8] C. Swindells, A. Unden, and T. Sang. Torquebar: an ungrounded haptic feedback device. In Proceedings of the 5th International Conference on Multimodal Interfaces, Vancouver, British Columbia, Canada, Nov. 2004.

[9] H. Z. Tan, M. A. Srinivasan, B. Eberman, and B. Cheng. Human factors for the design of force-reflecting haptic interfaces. In C. J. Radcliffe, editor, Proceedings of the Third International Symposium on Haptic Interfaces for Virtual Environment and Teleoperator Systems, ASME Dynamic Systems and Control, volume 55(1), pages 353-359. American Society of Mechanical Engineers, 1994.

[10] Y. Tanaka, J. Yamashita, and N. Nakamura. Mobile torque disply and haptic characteristics of human palm. In Proceedings of ICAT 2001, Tokyo, Japan, pages 115-120, Dec. 2001.

[11] H. Yano, M. Yoshie, and H. Iwata. Development of a non-grounded haptic interface using the gyro effect. In Proceedings of 11th International Symposium on Haptic Interfaces for Virtual Environment and Teleoperator Systems, pages 32-39, 2003. 